\title{
On spin scale-discretised wavelets on the sphere for the analysis of CMB polarisation
}

\author{
Jason D. McEwen ${ }^{1}$, Martin Büttner ${ }^{2}$, Boris Leistedt ${ }^{2}$, \\ Hiranya V. Peiris ${ }^{2}$, Pierre Vandergheynst ${ }^{3}$ and Yves Wiaux ${ }^{4}$ \\ ${ }^{1}$ Mullard Space Science Laboratory (MSSL), University College London (UCL), \\ Surrey RH5 6NT, UK \\ email: jason.mcewen@ucl.ac.uk \\ ${ }^{2}$ Department of Physics and Astronomy, University College London (UCL), \\ London WC1E 6BT, UK \\ email: \{martin.buettner.11, boris.leistedt.11, h.peiris\}@ucl.ac.uk \\ ${ }^{3}$ Institute of Electrical Engineering, Ecole Polytechnique Fédérale de Lausanne (EPFL), \\ CH-1015 Lausanne, Switzerland \\ email: pierre.vandergheynst@epfl.ch \\ ${ }^{4}$ Institute of Sensors, Signals \& Systems, Heriot-Watt University, \\ Edinburgh EH14 4AS, UK \\ email: y.wiaux@hw.ac.uk
}

\begin{abstract}
A new spin wavelet transform on the sphere is proposed to analyse the polarisation of the cosmic microwave background (CMB), a spin \pm 2 signal observed on the celestial sphere. The scalar directional scale-discretised wavelet transform on the sphere is extended to analyse signals of arbitrary spin. The resulting spin scale-discretised wavelet transform probes the directional intensity of spin signals. A procedure is presented using this new spin wavelet transform to recover E- and B-mode signals from partial-sky observations of CMB polarisation.
\end{abstract}

Keywords. cosmology: cosmic microwave background, cosmology: observations, cosmology: early universe, methods: data analysis, techniques: image processing

\section{Introduction}

The polarisation of the cosmic microwave background (CMB) is a powerful probe of the physics of inflation (Spergel \& Zaldarriaga 1997) and the reionisation history of the Universe (Zaldarriaga 1997). Numerous experiments have now measured CMB polarisation (some of the more recent include: Hanson et al. 2013; Naess et al. 2014; BICEP2 Collaboration 2014). Although the Planck satellite also measured CMB polarisation, polarisation data were not included in the Planck 2013 release (Planck Collaboration I 2013) but are anticipated later this year.

Since different physical processes often exhibit different symmetries, their signatures in observables like CMB polarisation may behave differently under a parity transform. CMB polarisation can be separated into parity even and parity odd components, so called Eand B-mode components, respectively (Zaldarriaga \& Seljak 1997). Density perturbations in the early Universe provide no mechanism to generate B-mode polarisation in the CMB, whereas gravitational waves can induce both E- and B-mode components. The detection of primordial B-mode polarisation would thus provide evidence for gravitational waves and would provide a powerful probe of the physics of inflation.

In these proceedings we outline a new spin wavelet transform on the sphere to analyse observations of CMB polarisation, a spin \pm 2 signal observed on the celestial sphere. In 
McEwen et al.

addition, we describe a simple technique based on this wavelet framework to separate Eand B-mode CMB polarisation components from partial-sky observations. We present a preliminary discussion only; further details of these methods, fast implementations, and a rigorous evaluation of their performance will be given in a series of forthcoming articles.

\section{Spin scale-discretised wavelets on the sphere}

Scalar wavelets on the sphere (e.g. Antoine \& Vandergheynst 1998, 1999; Baldi et al. 2009; McEwen et al. 2006a, 2007a, 2013; Marinucci et al. 2008; Narcowich et al. 2006; Starck et al. 2006; Wiaux et al. 2005, 2006, 2008; Leistedt et al. 2013) have proved an effective tool for analysing the temperature anisotropies of the CMB (e.g. Vielva et al. 2004; Vielva et al. 2006; McEwen et al. 2005, 2006b, 2008a, 2006c, 2007b, 2008b; Pietrobon et al. 2006; Faÿ et al. 2008; Feeney et al. 2011a,b; Bobin et al. 2013; Planck Collaboration XII 2013; Planck Collaboration XXIII 2013; Planck Collaboration XXIV 2014; Planck Collaboration XXV 2013). For a somewhat dated review see McEwen et al. (2007c). Spin wavelets to analyse the polarisation of the CMB have been constructed by Geller et al. (2008) and Starck et al. (2009). However, a spin wavelet transform on the sphere capable of probing the directional intensity of signals does not yet exist. $\dagger$ We propose such a transform here by extending the directional scale-discretised wavelet transform of Wiaux et al. (2008) to signals of arbitrary spin on the sphere.

Spin scale-discretised wavelets ${ }_{s} \Psi^{(j)} \in \mathrm{L}^{2}\left(\mathbb{S}^{2}\right)$ can be constructed on the sphere $\mathbb{S}^{2}$ in an analogous manner to the scalar wavelet construction (Wiaux et al. 2008; Leistedt et al. 2013; McEwen et al. 2013), that is simply by defining the spin harmonic coefficients of the wavelets in the factorised form:

$$
{ }_{s} \Psi_{\ell m}^{(j)} \equiv \kappa^{(j)}(\ell) \zeta_{\ell m}
$$

where ${ }_{s} \Psi_{\ell m}^{(j)}=\left\langle{ }_{s} \Psi^{(j)},{ }_{s} Y_{\ell m}\right\rangle$ are the spin $s \in \mathbb{Z}$ spherical harmonic coefficients of the wavelets, with ${ }_{s} Y_{\ell m}$ denoting the spherical harmonic functions and $\ell \in \mathbb{N}_{0}, m \in \mathbb{Z}$, such that $|s| \leqslant \ell$ and $|m| \leqslant \ell$. The kernel $\kappa^{(j)} \in \mathrm{L}^{2}\left(\mathbb{R}^{+}\right)$controls the angular localisation of the wavelets, while their directional properties are controlled by the directionality component $\zeta \in \mathrm{L}^{2}\left(\mathbb{S}^{2}\right)$, with harmonic coefficients $\zeta_{\ell m}=\left\langle\zeta, Y_{\ell m}\right\rangle$. The wavelet scale $j \in \mathbb{N}_{0}$ encodes the angular localisation of $\Psi^{(j)}$. The kernel and directionality component are defined as in the scalar setting (Wiaux et al. 2008; McEwen et al. 2013).

The wavelet transform of a spin signal ${ }_{s} f \in \mathrm{L}^{2}\left(\mathbb{S}^{2}\right)$ on the sphere is defined by the directional convolution of ${ }_{s} f$ with the wavelet ${ }_{s} \Psi^{(j)} \in \mathrm{L}^{2}\left(\mathbb{S}^{2}\right)$. The wavelet coefficients $W^{s} \Psi^{(j)} \in \mathrm{L}^{2}(\mathrm{SO}(3))$ thus read

$$
W^{s} \Psi^{(j)}(\rho) \equiv\left({ }_{s} f \star{ }_{s} \Psi^{(j)}\right)(\rho) \equiv\left\langle{ }_{s} f, \mathcal{R}_{\rho s} \Psi^{(j)}\right\rangle=\int_{\mathbb{S}^{2}} \mathrm{~d} \Omega(\omega)_{s} f(\omega)\left(\mathcal{R}_{\rho s} \Psi^{(j)}\right)^{*}(\omega),
$$

where $\omega=(\theta, \varphi) \in \mathbb{S}^{2}$ denotes spherical coordinates with colatitude $\theta \in[0, \pi]$ and longitude $\varphi \in[0,2 \pi), \mathrm{d} \Omega(\omega)=\sin \theta \mathrm{d} \theta \mathrm{d} \varphi$ is the usual rotation invariant measure on the sphere, and * denotes complex conjugation. The rotation operator is defined by

$$
\left(\mathcal{R}_{\rho s} \Psi^{(j)}\right) \equiv{ }_{s} \Psi^{(j)}\left(\mathbf{R}_{\rho}^{-1} \cdot \omega\right)
$$

where $\mathbf{R}_{\rho}$ is the three-dimensional rotation matrix corresponding to $\mathcal{R}_{\rho}$. Rotations are specified by elements of the rotation group $\mathrm{SO}(3)$, parameterised by the Euler angles

$\dagger$ Spin curvelets (Starck et al. 2009) could be used for a directional analysis however these are constructed on the base pixels of Healpix (Górski et al. 2005) and so do not live naturally on the sphere. 
$\rho=(\alpha, \beta, \gamma) \in \mathrm{SO}(3)$, with $\alpha \in[0,2 \pi), \beta \in[0, \pi]$ and $\gamma \in[0,2 \pi)$. Note that the wavelet coefficients are a scalar signal defined on the rotation group $\mathrm{SO}(3)$. The wavelet transform of Eqn. (2.2) thus probes the directional intensity of the signal of interest ${ }_{s} f$.

Provided the wavelets satisfy an admissibility property analogous to the scalar setting, the original signal can be synthesised exactly from its wavelet coefficients by

$$
{ }_{s} f(\omega)=\sum_{j=J_{0}}^{J} \int_{\mathrm{SO}(3)} \mathrm{d} \varrho(\rho) W^{s} \Psi^{j}(\rho)\left(\mathcal{R}_{\rho s} \Psi^{j}\right)(\omega),
$$

where $\mathrm{d} \varrho(\rho)=\sin \beta \mathrm{d} \alpha \mathrm{d} \beta \mathrm{d} \gamma$ is the usual invariant measure on $\mathrm{SO}(3)$ and $J_{0}$ and $J$ are the minimum and maximum wavelet scales considered, respectively, i.e. $J_{0} \leqslant j \leqslant J$. Throughout this description we have neglected to include a scaling function, which must be introduced to capture the low-frequency content of the analysed signal ${ }_{s} f$.

\section{E- and B-mode separation}

$\mathrm{CMB}$ experiments measure the scalar Stoke parameters $I, Q, U \in \mathrm{L}^{2}\left(\mathbb{S}^{2}\right)$, where $I$ encodes the intensity and $Q$ and $U$ the linear polarisation of the incident CMB radiation (the circular polarisation component of the four Stokes parameters $V \in \mathrm{L}^{2}\left(\mathbb{S}^{2}\right)$ is zero). The linear polarisation signal that is observed depends on the choice of local coordinate frame. The component $Q \pm \mathrm{i} U$ transforms under a rotation of the local coordinate frame by $\chi \in[0,2 \pi)$ as $(Q \pm \mathrm{i} U)^{\prime}(\omega)=\exp (\mp \mathrm{i} 2 \chi)(Q \pm \mathrm{i} U)(\omega)$ and is thus a spin \pm 2 signal on the sphere (Zaldarriaga \& Seljak 1997). The quantity $Q \pm \mathrm{i} U$ can be decomposed into parity even and odd components by $\tilde{E}(\omega)=-\frac{1}{2}\left[\bar{\partial}^{2}(Q+\mathrm{i} U)(\omega)+\partial^{2}(Q-\mathrm{i} U)(\omega)\right]$ and $\tilde{B}(\omega)=$ $\frac{\mathrm{i}}{2}\left[\bar{\partial}^{2}(Q+\mathrm{i} U)(\omega)-\partial^{2}(Q-\mathrm{i} U)(\omega)\right]$ respectively, where $\tilde{E}, \tilde{B} \in \mathrm{L}^{2}\left(\mathbb{S}^{2}\right)$ and $\check{\partial}$ and $\bar{\partial}$ are spin raising and lowering operators, respectively (Zaldarriaga \& Seljak 1997). Recovering Eand B-modes from full-sky observations is relatively straightforward, however in practice we observe the CMB over only part of the sky, since microwave emissions from our Galaxy obscure our view. A number of techniques have been developed to recover Eand B-modes from $Q$ and $U$ maps observed on the partial-sky (e.g. Lewis et al. 2002; Bunn et al. 2003; Kim 2011; Bowyer et al. 2011). Here we propose a simple alternative approach using the spin scale-discretised wavelet transform described above (a similar approach using needlets has been proposed by Geller et al. (2008), however there are some minor differences since spin needlets yield spin and not scalar wavelet coefficients).

First, consider the wavelet coefficients of the observable $Q+\mathrm{i} U$ signal computed by a spin wavelet transform: $W_{Q+\mathrm{i} U}^{2} \Psi^{(j)}(\rho) \equiv\left\langle Q+\mathrm{i} U, \mathcal{R}_{\rho 2} \Psi^{(j)}\right\rangle$. Second, consider the wavelet coefficients of the unobservable $\tilde{E}$ and $\tilde{B}$ signals computed by a scalar wavelet transform: $W_{\tilde{E}}^{\mathrm{o}^{\tilde{\Psi}^{j}}}(\rho) \equiv\left\langle\tilde{E}, \mathcal{R}_{\rho_{0}} \tilde{\Psi}^{j}\right\rangle$ and $W_{\tilde{B}}^{\mathrm{o}^{\tilde{\Psi}^{j}}}(\rho) \equiv\left\langle\tilde{B}, \mathcal{R}_{\rho_{0}} \tilde{\Psi}^{j}\right\rangle$. If the wavelet used in the scalar wavelet transform is a spin lowered version of the wavelet used in the spin wavelet transform, i.e. $\tilde{\Psi}^{j}=\bar{\delta}^{2}{ }_{2} \Psi^{j}$, then the wavelet coefficients of $\tilde{E}$ and $\tilde{B}$ are simply related to the wavelet coefficients of $Q+\mathrm{i} U$ by $W_{\tilde{E}}^{\mathrm{o} \tilde{\Psi}^{j}}(\rho)=-\operatorname{Re}\left[W_{Q+\mathrm{i} U}^{2} \Psi^{j}(\rho)\right]$ and $W_{\tilde{B}}^{0} \tilde{\Psi}^{j}(\rho)=-\operatorname{Im}\left[W_{Q+\mathrm{i} U}^{2 \Psi^{j}}(\rho)\right]$, respectively.

This leads to an elegant procedure to recover E- and B-modes from $Q$ and $U$ maps observed over the partial-sky. Firstly, compute the spin wavelet transform of $Q+\mathrm{i} U$. Secondly, mitigate the impact of the partial sky coverage in wavelet space, where signal content (and thus the influence of the mask) is localised in scale and position simultaneously. Thirdly, reconstruct $\tilde{E}$ and $\tilde{B}$ maps by inverse scalar wavelet transforms of the real and imaginary components, respectively, of the processed spin wavelet coefficients. 


\section{References}

Antoine, Vandergheynst, 1998, J. Math. Phys., 39, 8, 3987

Antoine, Vandergheynst, 1999, ACHA, 7, 1

Baldi, Kerkyacharian, Marinucci, Picard, 2009, Ann. Stat., 37 No.3, 1150, arXiv:math/0606599

BICEP2 Collaboration, 2014, submitted, arXiv:1403.3985

Bobin, Starck, Sureau, Basak, 2013, A\&A, 550, A73, arXiv:1206.1773

Bowyer, Jaffe, Novikov, 2011, arXiv, 1009, arXiv:1101.0520

Bunn, Zaldarriaga, Tegmark, de Oliveira-Costa, 2003, PRD, 67, 2, 023501, astro-ph/0207338

Faÿ, Guilloux, Betoule, Cardoso, Delabrouille, Le Jeune, 2008, PRD, 78, 8, 083013, arXiv:0807.1113

Feeney, Johnson, Mortlock, Peiris, 2011a, Phys. Rev. Lett., 107, 071301, 1012.1995

Feeney, Johnson, Mortlock, Peiris, 2011b, PRD, D84, 043507, 1012.3667

Geller, Hansen, Marinucci, Kerkyacharian, Picard, 2008, PRD, 78, 12, 123533, arXiv:0811. 2881

Górski, Hivon, Banday, Wandelt, Hansen, Reinecke, Bartelmann, 2005, ApJ, 622, 759, astro-ph/0409513

Hanson, et al., 2013, PRL, 111, 14, 141301, arXiv:1307.5830

Kim, 2011, A\&A, 531, A32, arXiv:1010.2636

Leistedt, McEwen, Vandergheynst, Wiaux, 2013, A\&A, 558, A128, 1, arXiv:1211.1680

Lewis, Challinor, Turok, 2002, PRD, 65, 2, 023505, astro-ph/0106536

Marinucci, et al., 2008, MNRAS, 383, 539, arXiv:0707.0844

McEwen, Hobson, Lasenby, 2006a, ArXiv, astro-ph/0609159

McEwen, Hobson, Lasenby, Mortlock, 2005, MNRAS, 359, 1583, astro-ph/0406604

McEwen, Hobson, Lasenby, Mortlock, 2006b, MNRAS, 371, L50, astro-ph/0604305

McEwen, Hobson, Lasenby, Mortlock, 2006c, MNRAS, 369, 1858, astro-ph/0510349

McEwen, Hobson, Lasenby, Mortlock, 2008a, MNRAS, 388, 2, 659, arXiv:0803.2157

McEwen, Hobson, Mortlock, Lasenby, 2007a, IEEE TSP, 55, 2, 520, astro-ph/0506308

McEwen, Vandergheynst, Wiaux, 2013, in SPIE Wavelets and Sparsity XV, arXiv:1308.5706

McEwen, Vielva, Hobson, Martínez-González, Lasenby, 2007b, MNRAS, 373, 1211, astro-ph/0602398

McEwen, Wiaux, Hobson, Vandergheynst, Lasenby, 2008b, MNRAS, 384, 4, 1289, arXiv:0704.0626

McEwen, et al., 2007c, JFAA, 13, 4, 495, arXiv:0704.3158

Naess, et al., 2014, ArXiv e-prints, arXiv:1405.5524

Narcowich, Petrushev, Ward, 2006, SIAM J. Math. Anal., 38, 2, 574

Pietrobon, Balbi, Marinucci, 2006, PRD, 74, 4, 043524, astro-ph/0606475

Planck Collaboration I, 2013, A\&A, in press, arXiv:1303.5062

Planck Collaboration XII, 2013, A\&A, in press, arXiv:1303.5072

Planck Collaboration XXIII, 2013, A\&A, in press, arXiv:1303.5083

Planck Collaboration XXIV, 2014, A\&A, in press, arXiv:1303.5084

Planck Collaboration XXV, 2013, A\&A, in press, arXiv:1303.5085

Spergel, Zaldarriaga, 1997, PRL, 79, 2180, astro-ph/9705182

Starck, Moudden, Bobin, 2009, A\&A, 497, 931, arXiv:0902.0574

Starck, Moudden, Abrial, Nguyen, 2006, A\&A, 446, 1191, astro-ph/0509883

Vielva, Martínez-González, Barreiro, Sanz, Cayón, 2004, ApJ, 609, 22, astro-ph/0310273

Vielva, Wiaux, Martínez-González, Vandergheynst, 2006, New Astronomy Review, 50, 880, astro-ph/0609147

Wiaux, Jacques, Vandergheynst, 2005, ApJ, 632, 15, astro-ph/0502486

Wiaux, Jacques, Vielva, Vandergheynst, 2006, ApJ, 652, 820, astro-ph/0508516

Wiaux, McEwen, Vandergheynst, Blanc, 2008, MNRAS, 388, 2, 770, arXiv:0712.3519

Zaldarriaga, 1997, PRD, 55, 1822, astro-ph/9608050

Zaldarriaga, Seljak, 1997, PRD, 55, 4, 1830, astro-ph/9609170 